\title{
Attitude Measurement of Special Aircraft Based on Geomagnetic and Angular Velocity Sensors
}

\author{
Zhang Pingan $(\mathbb{D}$, Wang Wei $(\mathbb{D}$, Gao Ming $(\mathbb{D}$, and Che Jinli $(\mathbb{1})$ \\ Shijiazhuang Campus of PLA Army Engineering University, Shijiazhuang 050003, China \\ Correspondence should be addressed to Zhang Pingan; 1213782904@qq.com
}

Received 1 September 2020; Revised 23 October 2020; Accepted 3 November 2020; Published 23 November 2020

Academic Editor: Jun-Wei Li

Copyright ( 2020 Zhang Pingan et al. This is an open access article distributed under the Creative Commons Attribution License, which permits unrestricted use, distribution, and reproduction in any medium, provided the original work is properly cited.

\begin{abstract}
Aiming at the problem of attitude test of special aircraft in flight, the combined test technology of geomagnetic sensor and angular velocity sensor is studied. The mathematical model of special aircraft roll attitude test based on combined measurement is established. The error models of special aircraft roll angle based on yaw angle input and pitch angle input are derived, respectively, and based on the actual flight trajectory data of aircraft, the mathematical model of special aircraft roll attitude test is established The simulation results show that the roll angle error input by yaw angle is between $-0.4^{\circ}$ and $0.9^{\circ}$, while the roll angle error input by pitch angle is between $-0.4^{\circ}$ and $1.2^{\circ}$, which shows that the calculation accuracy of roll angle input by yaw angle is higher, and the existence of magnetic measurement blind area is verified. In this paper, the method of judging the blind area of geomagnetic survey and the algorithm model of eliminating the influence of blind area are proposed.
\end{abstract}

\section{Introduction}

With the rapid development of military science and technology, the demand for precision guided special aircraft with long-range suppression, precision strike, high-efficiency damage, multipurpose, and multifunction is becoming more and more important in modern war. In order to reduce the high investment and high consumption of operational funds in the war, low-cost and trajectory correction aircraft have entered the research field $[1,2]$. It is an ideal way to realize equipment modernization and control the increase of armament cost that trajectory correction device is used to replace the traditional aircraft controller without replacing the launcher, and the accurate fire attack can be achieved by trajectory correction during flight [3]. In order to perform trajectory correction and guidance control functions, the position, velocity, and attitude angle information of special aircraft need to be determined. Attitude angle information includes pitch angle, yaw angle, and roll angle. Roll angle is the most significant and important information among attitude variables. According to the roll angle of the aircraft, the space position of the actuator can be determined, so as to realize the real-time correction and guidance control oper- ation of the actuator [4]. Therefore, the flight attitude measurement technology of special aircraft is one of the key technologies to realize flight path correction control [5-7].

At present, due to the harsh environment of aircraft attitude measurement and the limitation of single sensor, it is necessary to study a method of multisensor combination to measure aircraft attitude. At present, the integrated geomagnetic and satellite measurement system is more mature for the special aircraft attitude measurement. The special aircraft attitude angles measured by this system are trajectory inclination angle, trajectory deflection angle, and roll angle obtained by both. At present, geomagnetic and satellite integrated measurement system is more mature for attitude measurement of special aircraft. The attitude angles measured by this system are trajectory inclination angle, trajectory deflection angle, and roll angle obtained by both. However, in the process of flight route correction, the axis inclination angle and the roll angle obtained by both are needed; Zhang [8] and other scholars from Northwest China Institute of electrical and mechanical engineering have verified that there is a certain deviation between the trajectory angle and the axis angle through experiments, and the law of the deviation is related to the flight environment, so it is difficult to 
summarize the law. Lianyan [9] and Dandan [10] and other scholars have proposed some compensation methods for the magnetic measurement blind area, but the compensation method does not eliminate the influence of magnetic measurement blind area on attitude measurement. Therefore, this paper proposes a method of combining geomagnetic and angular velocity sensors to measure the attitude of special aircraft.

Since the impact acceleration of the special aircraft reaches $18000 \mathrm{~g}$ and the rolling speed reaches 300 revolutions per second, the angular velocity sensor cannot be used in this harsh environment. Therefore, in the design of flight path correction device, the impact battery is used to supply power to the control part, and the differential rudder is used to slow down the speed of the flight path correction part to start the angular velocity after reaching the test environment angular speed and geomagnetic sensors work. At present, the combination of angular velocity sensor and geomagnetic measurement system is often used in missiles, rockets, and ordinary aircrafts. Yun et al. [11] proposed an attitude algorithm about angular velocity and geomagnetic sensor, but in his paper, the feasibility of the algorithm is not analyzed. Zhu et al. [12] proposed an algorithm based on the difference quotient of single-axis gyroscope and geomagnetic sensor to measure the attitude of aircraft. However, in his research, the blind area of magnetic measurement in the process of attitude measurement was ignored. Ye et al. [13] used two-axis geomagnetism and three-axis gyroscopes to measure the flying attitude of missiles and arrows. The attitude calculation algorithm proposed by $\mathrm{Du}$ Ye achieved good results through experiments, but the influence of magnetic blind area measurement on attitude measurement was not considered in his research. Therefore, the main content of this paper is to measure the flight attitude of special aircraft in all directions and trajectory by using three-axis angular velocity sensor and three-axis geomagnetic sensor. The pitch angle and roll angle of the aircraft are measured by three-axis angular velocity sensor, and the other two attitude angles are measured by three-axis geomagnetic sensor. The simulation results show that the results show that the yaw angle input geomagnetic sensor is more effective in measuring the attitude of aircraft, and an effective method to solve the blind area of magnetic measurement is proposed.

\section{Configuration and Measurement Principle of Geomagnetic and Angular Velocity Sensors}

2.1. Two Installation Methods of Sensors. It is assumed that the center of gravity of the special aircraft is on the axis, and the $X_{\mathrm{d}}$ axis of the aircraft coordinate system is located along the axis. As shown in Figure 1, the $X_{c}$ axis of the geomagnetic sensor coincides with the aircraft axis, and the other two axes are, respectively, positioned along the $Y_{\mathrm{d}}$ and $Z_{\mathrm{d}}$ axes of the aircraft coordinate system. The sensitive axis configuration of the angular velocity sensor is completely parallel to the three axes of the geomagnetic sensor, and the installation position is on the aircraft axis.

Among them, the aircraft coordinate system is represented by $o X_{\mathrm{d}} Y_{\mathrm{d}} Z_{\mathrm{d}}$, the geomagnetic sensor coordinate system is represented by $o X_{c} Y_{c} Z_{c}$, and the angular velocity sensor coordinate system is represented by $o_{1} X_{\mathrm{r}} Y_{\mathrm{r}} Z_{\mathrm{r}}$.

2.2. Principle of Combined Measurement. The ground datum coordinate system adopts the north-sky-east coordinate system. The north axis is represented by N, the sky axis is represented by $\mathrm{S}$, and the east axis is represented by $\mathrm{E}$. the north and east axes are in the horizontal plane. The International Geomagnetic Reference Field (IGRF) can be used to obtain the magnetic dip angle and magnetic declination angle of geomagnetic vector in the north-sky-east coordinate system, which are set as $I$ and $D$; thus, the component of the geomagnetic vector in the three axes of the north-sky-east coordinate system can be obtained:

$$
\left[\begin{array}{lll}
B_{\mathrm{N}} & B_{\mathrm{S}} & B_{\mathrm{E}}
\end{array}\right]=\left[\begin{array}{lll}
B \cos I \cos D & B \sin I \quad B \cos I \sin D
\end{array}\right],
$$

where $B$ is the geomagnetic field strength; $B_{\mathrm{N}}, B_{\mathrm{S}}$, and $B_{\mathrm{E}}$ are the three axis components of the geomagnetic vector in the north-sky-east coordinate system. The direction of sensitive axis of geomagnetic sensor is consistent with that of aircraft coordinate system. In the flight process of special aircraft, the relationship between geomagnetic and aircraft attitude angle can be obtained by real-time measurement of geomagnetic data by the geomagnetic measurement unit loaded on the aircraft, as shown in the following formula.

$$
\left\{\begin{array}{l}
B_{x c}=B \cos I \cos \alpha \cos (D+\beta)+B \sin I \sin \alpha, \\
B_{y c}=-B \cos I \sin \alpha \cos \gamma \cos (D+\beta)+B \cos I \sin \gamma \sin (D+\beta)+B \sin I \cos \alpha \cos \gamma, \\
B_{z c}=B \cos I \sin \alpha \sin \gamma \cos (D+\beta)+B \cos I \cos \gamma \sin (D+\beta)-B \sin I \cos \alpha \sin \gamma .
\end{array}\right.
$$

In the above formula, $\alpha, \beta, \gamma$ are the pitch angle, yaw angle, and roll angle obtained from the north-sky-east coordinate system to the aircraft coordinate system according to the $Y, Z$, and $X$ axis sequence. $B_{x c}, B_{y c}$, and $B_{z c}$, respectively, represent the components of geomagnetic vector on the three axes of the coordinate system $o X_{c} Y_{c} Z_{c}$, which can be 


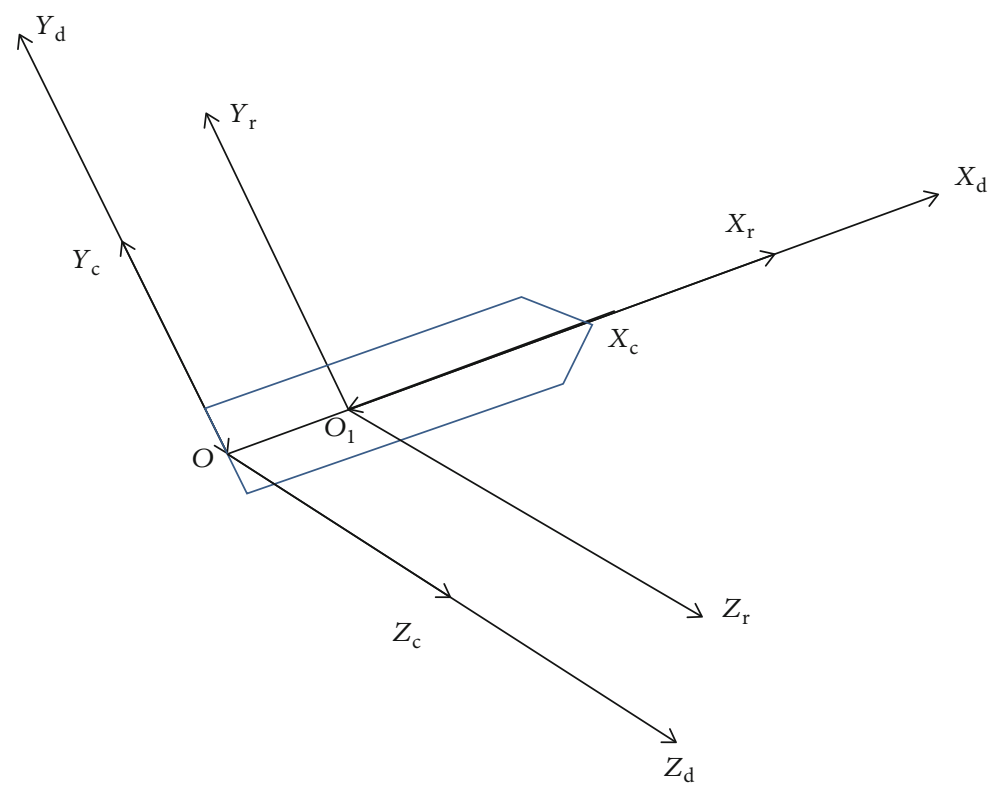

FIGURE 1: Installation method of sensors.

calculated by the output value of the three-axis geomagnetic sensor. It is shown in the following formula.

$$
\left\{\begin{array}{l}
B_{X C}=\frac{V_{X C}-V_{X C 90}}{(S K)_{X C}}, \\
B_{Y C}=\frac{V_{Y C}-V_{Y C 90}}{(S K)_{Y C}}, \\
B_{Z C}=\frac{V_{Z C}-V_{Z C 90}}{(S K)_{Z C}} .
\end{array}\right.
$$

In the above formula, $V_{X C}, V_{Y C}$, and $V_{Z C}$ are output voltage of three axis geomagnetic sensor; $V_{X C 90}, V_{Y C 90}$, and $V_{Z C 90}$ are zero output value of three axis geomagnetic sensor; $(\mathrm{SK}) X_{\mathrm{C}},(\mathrm{SK}) Y_{\mathrm{C}}$, and $(\mathrm{SK}) Z_{\mathrm{C}}$ are the product of sensitivity and circuit amplification factor of three axis geomagnetic sensor.

Angular velocity sensor can get the angular velocity vector of aircraft coordinate system relative to north-sky-east coordinate system as follows $\omega=(p, q, r)$, where $p$ is the angular velocity of $X_{\mathrm{d}}$ and $Z_{\mathrm{d}}$ axes relative to $Y_{\mathrm{d}}$ axis (left rotation is positive), $q$ is angular velocity of $X_{\mathrm{d}}$ and $Y_{\mathrm{d}}$ axes relative to $Z_{\mathrm{d}}$ axis (topspin is positive), and $R$ is angular velocity of $Y_{\mathrm{d}}$ and $Z_{\mathrm{d}}$ axes relative to $X_{\mathrm{d}}$ axis (right helix is positive). Angular velocity vector and attitude angular velocity of aircraft can be transformed through the following equations:

$$
\left[\begin{array}{c}
\dot{\alpha} \\
\dot{\beta} \\
\dot{\gamma}
\end{array}\right]=\left[\begin{array}{ccc}
\cos \gamma & -\sin \gamma & 0 \\
\frac{\sin \gamma}{\cos \alpha} & \frac{\cos \gamma}{\cos \alpha} & 0 \\
\sin \gamma \tan \alpha & \cos \gamma \tan \alpha & 1
\end{array}\right]\left[\begin{array}{l}
q \\
p \\
r
\end{array}\right] .
$$

In the above formula, $\dot{\alpha}, \dot{\beta}$, and $\dot{\gamma}$ are the pitch angular velocity, yaw angular velocity, and roll angular velocity of the special aircraft. From the above formula, the calculation formula of yaw angular velocity and pitch angular velocity can be obtained as follows:

$$
\begin{aligned}
& \dot{\beta}(t)=q(t) \frac{\sin \gamma(t)}{\cos \alpha(t)}+p(t) \frac{\cos \gamma(t)}{\cos \alpha(t)}, \\
& \dot{\alpha}(t)=q(t) \cos \gamma(t)-p(t) \sin \gamma(t) .
\end{aligned}
$$

2.2.1. Input Geomagnetic Sensor with Yaw Angle. The yaw angle of the aircraft can be obtained by numerical integration of the yaw rate, as shown in the following formula.

$$
\beta_{y}(t)=\beta_{y}\left(t_{\mathrm{i}}\right)+\int_{t_{\mathrm{i}}}^{t}[\dot{\beta}(t)] d t .
$$

In the above formula, $t_{\mathrm{i}}$ is the launch time of the aircraft or the next data update time, and $\beta_{y}\left(t_{\mathrm{i}}\right)$ is the yaw angle of the aircraft at the initial time or the next data update time. The pitch angle can be calculated by introducing the yaw angle obtained from the above formula into "(2)". The mathematical model is shown in the following formula.

$$
\alpha_{y}=\arcsin \frac{B_{x c}}{B \sqrt{\cos ^{2} I \cos ^{2}\left(D+\beta_{y}\right)+\sin ^{2} I}}-\operatorname{arctg} \frac{\cos \left(D+\beta_{y}\right)}{\tan I} .
$$

The mathematical model of roll angle can be obtained by introducing the pitch angle and yaw angle obtained from the above formula into "(2)," as shown in the following formula.

$$
\gamma_{y}=\operatorname{arctg} \frac{B_{z y} B_{y c}-B_{y y} B_{z c}}{B_{z y} B_{z c}+B_{y y} B_{y c}},
$$




$$
\left\{\begin{array}{l}
B_{z y}=B \cos I \sin \left(D+\beta_{y}\right) \\
B_{y y}=-B \cos I \sin \alpha_{y} \cos \left(D+\beta_{y}\right)+B \sin I \cos \alpha_{y} .
\end{array}\right.
$$

2.2.2. Input Geomagnetic Sensor with Pitch Angle. The pitch angle of the aircraft can be obtained by numerical integration of the pitch angular velocity, as shown in the following formula:

$$
\alpha_{p}(t)=\alpha_{p}\left(t_{\mathrm{i}}\right)+\int_{t_{\mathrm{i}}}^{t} \dot{\alpha}(t) d t
$$

In the above formula, $\alpha_{p}\left(t_{\mathrm{i}}\right)$ is the pitch angle of the aircraft at the initial time or the next data update time. The mathematical model of yaw angle and roll angle can be obtained by introducing pitching angle into "(2)," as shown in the following formula:

$$
\begin{aligned}
& \beta_{p}(t)=\arccos \frac{B_{x c}-B \sin I \sin \alpha_{p}(t)}{B \cos I \cos \alpha_{p}(t)}-D, \\
& \gamma_{p}=\operatorname{arctg} \frac{B_{z p} B_{y c}-B_{y p} B_{z c}}{B_{z p} B_{z c}+B_{y p} B_{y c}}, \\
& \left\{\begin{array}{l}
B_{z p}=B \cos I \sin \left(D+\beta_{p}\right), \\
B_{y p}=-B \cos I \sin \alpha_{p} \cos \left(D+\beta_{p}\right)+B \sin I \cos \alpha_{p} .
\end{array}\right.
\end{aligned}
$$

In this section, the installation mode of geomagnetic and angular velocity sensors on special aircraft is explained, and the mathematical models of attitude angle measurement with yaw angle input or pitch angle input are established.

\section{Error Analysis of Combined Measurement}

3.1. Analysis of Blind Area in Magnetic Measurement. As the basic energy field of the earth, geomagnetic field is an important inherent resource on the earth. The geomagnetic intensity at any point on the earth surface is determined by the geomagnetic elements, which provides a good reference for geomagnetic navigation. According to the longitude, latitude, and altitude in the flight process of special aircraft, the magnetic inclination angle and magnetic declination angle of geomagnetic vector in the north-sky-east coordinate system can be obtained through the geomagnetic model, so as to obtain the components of geomagnetic vector on the three axes of north-sky-east coordinate system: $B_{\mathrm{N}}, B_{\mathrm{S}}$, and $B_{\mathrm{E}}$. Combined with the output value of geomagnetic and angular velocity sensor, the roll angle of aircraft can be calculated. It is shown as follows:

$$
\sin \gamma=\frac{B_{z} B_{y c}+B_{y} B_{z c}}{B_{y c}^{2}+B_{z c}^{2}}
$$

$$
\left\{\begin{array}{l}
B_{z}=B \cos I \sin (D+\beta), \\
B_{y}=-B \cos I \sin \alpha \cos (D+\beta)+B \sin I \cos \alpha .
\end{array}\right.
$$

It can be seen from "(14)" that when $B_{y c}=B_{z c}=0$, singular values will be generated. Since the range of pitch angle and yaw angle is $(-\pi / 2, \pi / 2)$, the following formula can be obtained:

$$
\begin{aligned}
& \left\{\begin{array}{l}
\alpha=I, \\
\beta=-D,
\end{array}\right. \\
& \left\{\begin{array}{l}
\alpha=-I, \\
\beta=\pi-D .
\end{array}\right.
\end{aligned}
$$

It can be seen from the above formula that when the aircraft axis is close to parallel to the geomagnetic vector, that is, when the output of $X_{C}$ of geomagnetic sensor is maximum, the output of $Y_{\mathrm{C}}$ axis and $Z_{\mathrm{C}}$ axis of geomagnetic sensor only has noise and zero drift value. At this time, the roll angle of aircraft cannot be obtained. Therefore, the $X_{\mathrm{C}}$ output of the geomagnetic sensor is close to the maximum value in the small range with the geomagnetic vector as the center. According to "(8)," the roll angle cannot be calculated or the error is large. This small range is called magnetic measurement blind area. When the aircraft enters the blind area of magnetic measurement, there will be a large error when using the geomagnetic sensor to calculate the roll angle. Therefore, the angular velocity sensor can temporarily replace the geomagnetic sensor to measure the special aircraft roll angle. The yaw rate and roll rate of the aircraft can be calculated by "(4)." The mathematical model is expressed as follows:

$$
\left\{\begin{array}{l}
\dot{\beta}(t)=\frac{q(t) \sin \gamma(t)+p(t) \cos \gamma(t)}{\cos \alpha(t)}, \\
\dot{\gamma}(t)=q(t) \sin \gamma(t) \tan \alpha(t)+p(t) \cos \gamma(t) \tan \alpha(t)+r(t) .
\end{array}\right.
$$

The yaw angle and roll angle can be obtained by integrating the yaw rate and roll rate with time, respectively, as shown in the following formula:

$$
\left\{\begin{array}{l}
\beta(t)=\beta\left(t_{\mathrm{j}}\right)+\int_{t_{\mathrm{j}}}^{t} \dot{\beta}(t) d t \\
\gamma(t)=\gamma\left(t_{\mathrm{j}}\right)+\int_{t_{\mathrm{j}}}^{t} \dot{\gamma}(t) d t
\end{array}\right.
$$

In the above formula, $t_{\mathrm{j}}$ represents the time when the special aircraft enters the blind zone of magnetic measurement; $\beta\left(t_{\mathrm{j}}\right)$ and $\gamma\left(t_{\mathrm{j}}\right)$ are the yaw angle and roll angle when the special aircraft enters the blind area of magnetic measurement.

3.2. Error Analysis of Yaw Angle Input. In order to study the influence of yaw angle variation on the measurement of pitch 
TABLE 1: Simulation parameters.

\begin{tabular}{lcccc}
\hline Flight time (s) & Sampling interval $(\mathrm{s})$ & Initial pitch angle $\left(^{\circ}\right)$ & Initial yaw angle $\left(^{\circ}\right)$ & Initial flight speed $(\mathrm{m} / \mathrm{s})$ \\
\hline 87.5 & 0.1 & 45 & 75 & 897
\end{tabular}

Flight distance (m) Highest position of flight path (m) Magnetic inclination $\left({ }^{\circ}\right)$ Magnetic declination $\left({ }^{\circ}\right)$ Magnetic field intensity (nT) 27126.4 9377 $-48.17^{\circ}$ $-2.32^{\circ}$ 50986.1

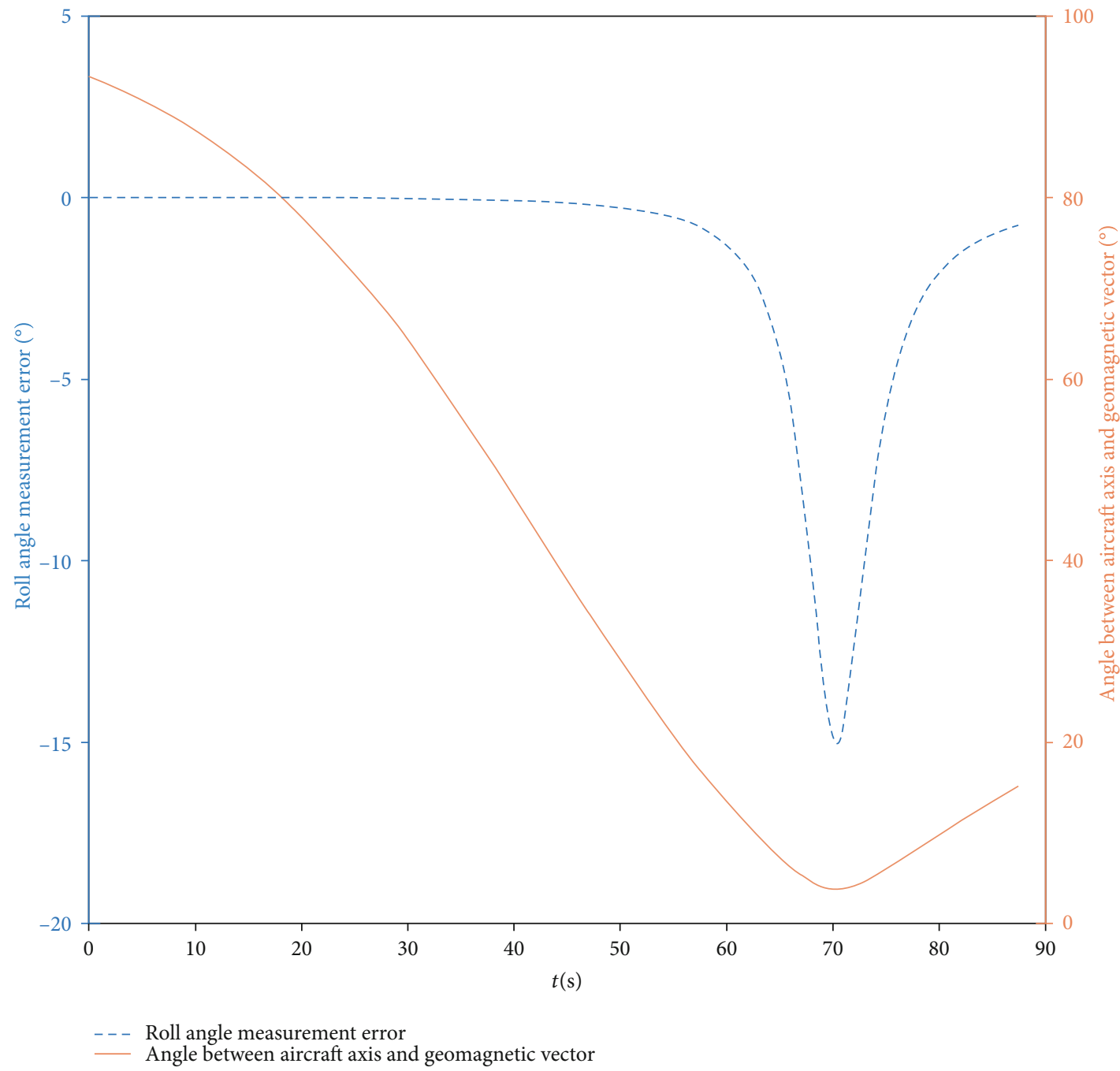

Figure 2: Comparison of roll angle error and angle change between aircraft axis and geomagnetic field.

angle and roll angle, the following formula is obtained by deriving the pitch angle in "(6)":

$$
\begin{aligned}
d \alpha_{y}= & {\left[\frac{B_{x c} \cos ^{2} I \sin \left[2\left(D+\beta_{y}\right)\right]}{2 \sqrt{B^{2}\left[\cos ^{2} I \cos ^{2}\left(D+\beta_{y}\right)+\sin ^{2} I\right]-B_{x c}^{2}}}\right.} \\
& \left.+\frac{\tan I \sin \left(D+\beta_{y}\right)}{\tan ^{2} I+\cos ^{2}\left(D+\beta_{y}\right)}\right] d \beta_{y} .
\end{aligned}
$$

By deriving the roll angle in "(8)," the following formula can be obtained:

$$
\begin{aligned}
& d \gamma_{y}= \frac{\left(\partial B_{z y} / \partial \beta_{y}\right) B_{y y}-\left(\left(\partial B_{y y} / \partial \alpha_{y}\right)\left(d \alpha_{y} / d \beta_{y}\right)+\left(\partial B_{y y} / \partial \beta_{y}\right)\right) B_{z y}}{B_{z y}^{2}+B_{y y}^{2}} d \beta_{y}, \\
&\left\{\begin{array}{l}
\frac{\partial B_{y y}}{\partial \alpha_{y}}=-B \cos I \cos \alpha_{y} \cos \left(D+\beta_{y}\right)-B \sin I \sin \alpha_{y}, \\
\frac{\partial B_{y y}}{\partial \beta_{y}}=B \cos I \sin \alpha_{y} \sin \left(D+\beta_{y}\right), \\
\frac{\partial B_{z y}}{\partial \beta_{y}}=B \cos I \cos \left(D+\beta_{y}\right) .
\end{array}\right.
\end{aligned}
$$

3.3. Error Analysis of Pitch Angle Input. In order to study the influence of pitch angle variation on the calculation of yaw 


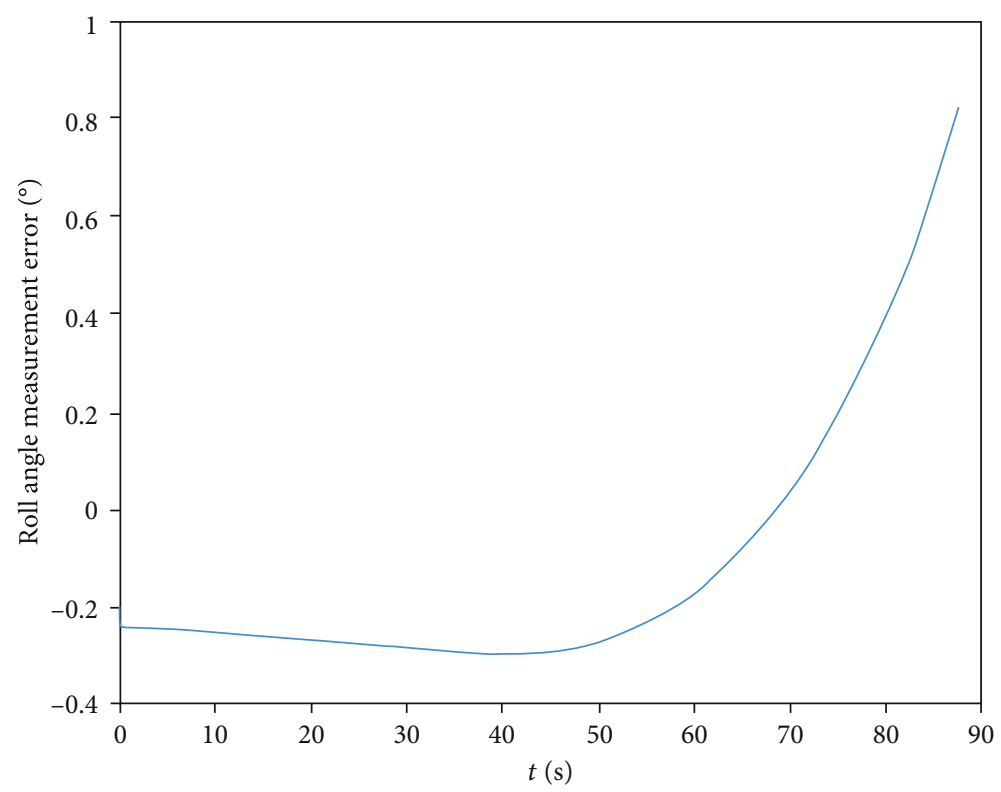

FIgURE 3: Roll angle error input with yaw angle.

angle, the following formula is obtained by deriving the yaw angle in "(11)":
By deriving the roll angle in "(12)," the following formula can be obtained:

$d \beta_{p}=\frac{B \sin I-B_{x c} \sin \alpha_{p}}{B \cos I \cos \alpha_{p} \sqrt{\left(B \cos I \cos \alpha_{p}\right)^{2}-\left(B_{x c}-B \sin I \sin \alpha_{p}\right) s^{2}}} d \alpha_{p}$.

$$
\begin{gathered}
d \gamma_{p}=\frac{-\left(\partial B_{y p} / \partial \beta_{p}\right)\left(d \beta_{p} / d \alpha_{p}\right) B_{z p}-\left(\partial B_{y p} / \partial \alpha_{p}\right) B_{z p}+\left(\partial B_{z p} / \partial \beta_{p}\right)\left(d \beta_{p} / d \alpha_{p}\right) B_{y p}}{B_{z p}^{2}+B_{y p}^{2}} d \alpha_{p}, \\
\left\{\begin{array}{l}
\frac{\partial B_{y p}}{\partial \alpha_{p}}=-B \cos I \cos \alpha_{p} \cos \left(D+\beta_{p}\right)-B \sin I \sin \alpha_{p}, \\
\frac{\partial B_{y p}}{\partial \beta_{p}}=B \cos I \sin \alpha_{p} \sin \left(D+\beta_{p}\right), \\
\frac{\partial B_{z p}}{\partial \beta_{p}}=B \cos I \cos \left(D+\beta_{p}\right) .
\end{array}\right.
\end{gathered}
$$

In this section, the range center of magnetic measurement blind area is determined, and the idea of using angular velocity sensor instead of geomagnetic sensor to measure the attitude angle of special aircraft in magnetic measurement blind area is proposed. The attitude measurement of all trajectory and omni-directional special aircraft is realized. The error of pitch angle and yaw angle measured by angular velocity sensor leads to the deviation of roll angle calculation. Therefore, the mathematical model of roll angle error with pitch angle as variable and yaw angle as variable is estab- lished, which lays a foundation for the feasibility of simulation verification in the next step.

\section{Simulation Verification}

In order to analyze the error of special aircraft attitude measurement based on the combination of Geomagnetism and angular velocity sensor, this paper selects certain place in Chengdu $\left(30.67^{\circ} \mathrm{N}, 104.07^{\circ} \mathrm{E}\right)$ as an example. Based on the latest IGRF given by the International Association of 


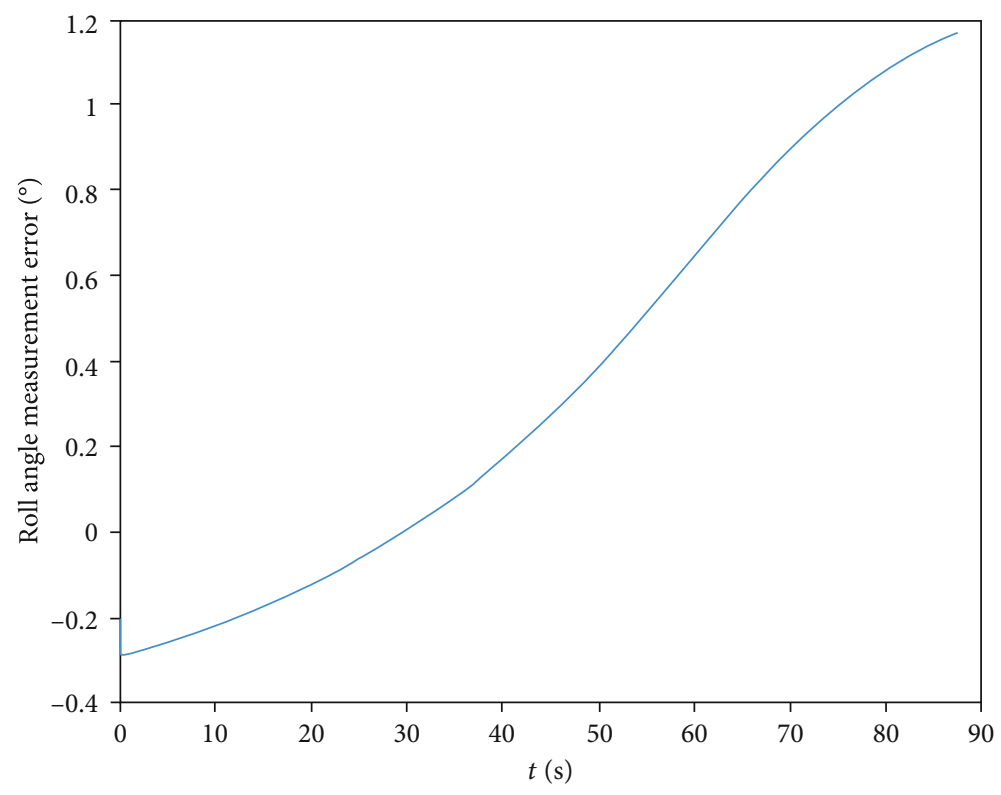

FIgURe 4: Roll angle error input with pitch angle.

Geomagnetism and Aeronomy, the local geomagnetic intensity, as well as the magnetic inclination and magnetic declination of geomagnetic vector in the North-Sky-East coordinate system, is obtained. The above measurement algorithm is simulated in MATLAB by using the actual trajectory data of special aircraft. The specific simulation parameters are shown in Table 1. The simulation results are shown in Figure 2 when launching with pitch angle of $45^{\circ}$ and yaw angle of $0^{\circ}$, and the angle change between special aircraft axis and geomagnetic vector and the measurement error of special aircraft rolling attitude are compared to verify the real existence of magnetic measurement blind area and the geomagnetic sensor in magnetic measurement In the blind area, the measurement error of roll angle is large.

Figures 3 and 4 are simulated with the initial yaw angle of $75^{\circ}$. It can be seen from Figure 3 that the angular velocity sensor is used to measure the yaw angle of the special aircraft, and then the roll angle calculated by the geomagnetic sensor is input. The error value is between $-0.4^{\circ}$ and $0.9^{\circ}$. It can be seen from Figure 4 that the pitch angle of the special aircraft is measured by the angular velocity sensor, and then, the roll angle is obtained by using the geomagnetic sensor. The error value is between $-0.4^{\circ}$ and $1.2^{\circ}$. In comparison, the error of the attitude of the aircraft is small when the yaw angle is input into the geomagnetic sensor. At the same time, the feasibility of the algorithm is verified.

\section{Conclusion}

According to the principle of the mathematical model, the roll angle error equations with yaw angle input and pitch angle input are derived, respectively. At the same time, through the analysis of the calculation process of roll angle, the center of magnetic measurement blind area is calculated. By using the flight trajectory data of special aircraft to establish a simulation model in MATLAB, the existence of mag- netic measurement blind area is verified, and whether the special aircraft exists in the magnetic measurement blind area can be judged by calculating the angle between the elastic axis and the geomagnetic vector. At the same time, it is proposed that the angular velocity sensor can replace the geomagnetic sensor in the blind area to measure the roll angle of the aircraft. Through the error model and flight trajectory data simulation, it is calculated that the roll angle measurement error will be between $-0.4^{\circ}$ and $0.9^{\circ}$ when the yaw angle is input, and the roll angle measurement error will be between $-0.4^{\circ}$ and $1.2^{\circ}$ when the pitch angle is input. In this paper, the algorithm of measuring special aircraft flight attitude by combining angular velocity sensor and geomagnetic sensor is studied, and the influence of magnetic measurement blind area on measurement result is effectively avoided, and the full range and full trajectory measurement of aircraft attitude is realized. Our future work will focus on the optimization of attitude measurement algorithm and precise navigation control of aircraft.

\section{Data Availability}

The data used to support the findings of this study are available from the corresponding author upon request.

\section{Conflicts of Interest}

The authors declare that they have no conflicts of interest.

\section{References}

[1] B. Jiefeng, Projectile Image Finishing Technology Based on Combined Measurement, Nanjing University of Technology, 2008. 
[2] Z. Minquan, L. Dongfang, W. Dongmei, and P. Yanke, "A review of the development of ballistic correction projectile," Acta Armamentarii, vol. 2010, no. S2, pp. 127-130, 2010.

[3] D. Storsved, "PGK and the impact of affordable precision on the fires mission," in 43rd Annual Guns \& Missiles Symposium, New Orleans, 2008.

[4] C. Yongwei, Research on Key Technologies of Projectile Roll Angle Identification System Based on Geomagnetic Detection, Nanjing University of Science and Technology, 2007.

[5] H. S. Lee, K. Kim, H. Y. Park, G. P. Chan, and J. G. Lee, "Roll estimation of a smart munition using a magnetometer based on an unscented Kalman filter," in Aiaa Guidance, Navigation \& Control Conference \& Exhibit, Honolulu, Hawaii, August 2013.

[6] G. Yijin, Roll Angle Measurement of Rotationally Stabilized Projectile Based on Magnetoresistive Sensor, Beijing University of Technology, 2015.

[7] C. A. O. Peng, Y. Ji-yan, W. Xiao-ming, Y. Wen-jin, and W. U. You-long, "High-frequency measurement and calculation study of systematic errors of high-rolling projectile roll anglebased on a combination of MR/GNSS," Acta Armamentarii, vol. 35, no. 6, pp. 795-800, 2014.

[8] L. Zhang, Z. Q. Zhao, B. N. Fan, and Y. Zhang, "Analysis on coning motion of high-speed spin-stabilized projectile base on on-board magnetic test," Acta Armamentarii, vol. 37, no. 12, pp. 2235-2241, 2016.

[9] S. Lianyan, Y. Shuxing, and Z. Xiaqing, "Application analysis of Mr / GPS guidance in rotary rocket," Journal of Missile, Arrow and Guidance, vol. 26, no. 2, pp. 1145-1147, 2006.

[10] Y. Dandan, L. Xinhua, Y. Wenjun, and G. Jun, "Method of roll attitude determination based on GPS and magneto-resistive sensor," Systems Engineering and Electronics, vol. 40, no. 11, article 2512, 2018.

[11] W. Yun, X. Changfan, and C. Ming, "The school study on attitude measurement of projectile body based on magnetic and angular sensors," Journal of Projectiles, Rockets, Missiles and Guidance, vol. 2012, no. 5, pp. 28-30, 2012.

[12] X. Zhu, Q. Jia, Y. Zhao, J. Song, and Y. He, “Attitude measurement method of geomagnetic/gyro combination based on difference-quotient," in 2020 IEEE 4th Information Technology, Networking, Electronic and Automation Control Conference (ITNEC), Chongqing, China, June 2020.

[13] D. Ye, F. Shun-shan, Y. Da-wei, A. Jing, and Z. Yi, "An attitude determination system using magnetometers and MEMS gyro," Transactions of Beijing Institute of Technology, vol. 34, no. 12, 2014. 